\title{
Survey on Wireless Sensor Routing Protocols
}

\author{
Abhishek Kumar Pandey ${ }^{1}$, Samir Srivastava ${ }^{2}$ \\ ${ }^{1}$ M.Tech Scholar, Computer Science \& Engineering, KNIT, Sultanpur, (U.P) India \\ ${ }^{2}$ Associate Professor, Computer Science \& Engineering, KNIT, Sultanpur, (U.P) India
}

\begin{abstract}
Wireless sensor networks is a group of sensor nodes which works jointly to perform a common application, it consists of low cost, low power, small in size and multi functional sensor nodes. They are densely deployed in the field. Because sensors are operated by battery power life time of the wireless nodes are limited. So we need some efficient routing techniques by which we can save the life time of the senor node. In this paper we discuss the different types of routing protocol. Routing protocols are application dependent based on the network structure we can classify the routing techniques in three categories: data centric, hierarchical and location base. This survey paper is a base paper to understand WSN routing techniques its merits and demerits so one can easily identify routing protocol for specific application.
\end{abstract}

Keywords: Wireless Sensor Networks, Challenges, Energy Efficient Protocols, Data-Centric protocols, Hierarchical Protocols and Location Based Protocols.

\section{Introduction}

A data network or computer network is a combination of computer coupled to each other for the sharing of exchange resources [1]. Wireless sensor network is the network of hundreds or thousands of sensor nodes also known as motes. Processing capability, storage and battery power is limited in the sensor node [2]. In this network, node senses the data and sends it to the base station. Then it analyzes the received data and computation is performed on received data to the base station, which gives the human understandable result. The sink is having unlimited battery power. The basic idea behind WSNs is that, while the capability of each individual sensor node is limited, the aggregate power of the entire network is sufficient for the required mission.

\section{Application}

Wireless sensor network are having many application, some of them are real time and some of them are non real time. Weather monitoring, intrusion detection, target detection in battle field, disaster management, and biomedical field are some of the applications of wireless sensor network Environmental monitoring of water and soil. Coordinated vehicle tracking.

\section{Challenges}

Designing of the routing protocol for wireless sensor network contains challenging factors, some challenges are discuss bellow [3].

(a) Sensor nodes are randomly deployed, so the implementation of the routing protocol is done in such a manner that entire nodes and base station may remain in automatic connectivity mode.

(b) Since, the nodes are having very less battery power, so our motive should be to save the energy of battery, hence, we should develop such routing protocol which consumes very less energy during transmission and accuracy should be very high. (c) Sensor node has very less computation capability. So it cannot support very complex routing protocol, so routing protocol should be very simple and light weight.

(d) One of the most design issue is fault tolerance [4], as we know that sensor nodes having very less energy so, Nodes die fast, so routing protocol is such that all of the nodes sending the data from redundant path.

(e) Network should be scalable, Hence routing protocol is such that, when the topology or size of network changes, it should not be affected by the operation of network.

(f) Sensor network contains some heterogeneity of nodes in term of energy, which is affected to the operation, so routing protocol should consider the node heterogeneity.

\section{Characteristics}

The nodes of the wireless sensor network are having some characteristic which are given below:-

(a) The size of the node is so small and less scalable.

(b) Node has less storage capacity.

(c) The resources for the node are limited.

(d) Sensor nodes are operated by some batteries, which are having very less power.

\section{Different Routing Protocols in wireless sensor networks}

The routing protocols in WSNs can be classified into datacentric, hierarchical, location based routing depending on the network structure as shown in figure 1 . In data-centric, all the nodes are functionally equivalent and associate in routing a query received from the base station to the event. In hierarchical approach, some nodes have added responsibilities in order to reduce the load on other nodes in the network. In location based, the knowledge of positions of sensor nodes is exploited to route the query from the base station to the event.

a) Data Centric Routing Protocol

b) Hierarchical Routing Protocol

c) Location- Base Routing Protocol 


\section{International Journal of Science and Research (IJSR)}

ISSN (Online): 2319-7064

Index Copernicus Value (2013): 6.14 | Impact Factor (2015): 6.391

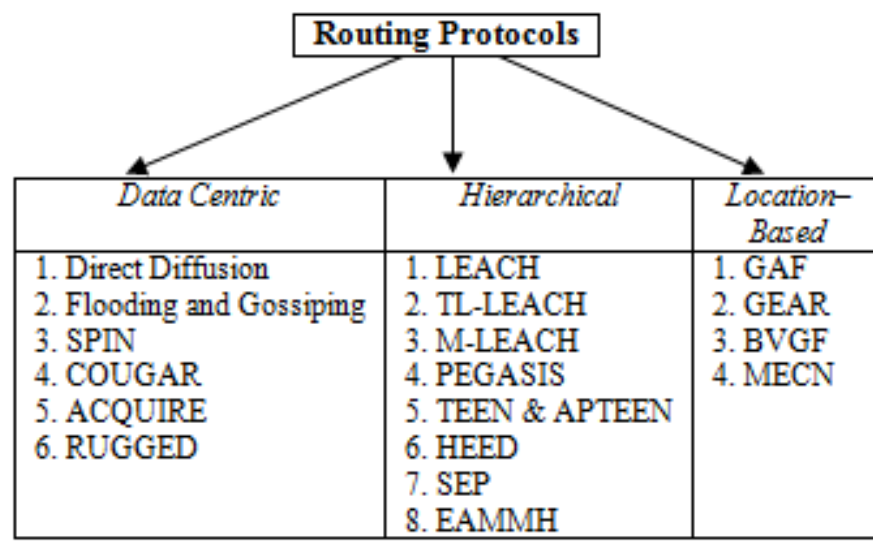

Figure 1: Categorization of routing Protocols

\subsection{Data Centric Protocol}

Many applications of wireless sensor networks involve nodes to be densely and randomly deployed. This makes it very difficult to assign global identifier to each node. Users show interest in querying a specific area rather than a single node. In the wireless sensor networks, traditional address-based networks routing protocols use more energy consumption and are inefficient as WSN routing protocols.

In data-centric routing protocol, the sink node sends request to the particular region and waits for the response from the sensor nodes which are located in this preferred region. For data- centric characteristics of sensor queries, an attributebased naming scheme is used where each node of the same attribute plays the same role.

\subsubsection{Direct Diffusion}

The direct diffusion method is proposed by Intanagonwiwat et al [19]. In this method all the nodes senses data and all of the data is aggregated in a central node where the redundancy of the node is removed and finally data are transmitted to the base station. This method reduces the number of transmission and energy of network is saved as well as the network life time is increases.

\subsubsection{Flooding and Gossiping}

There are two classical mechanisms Flooding and Gossiping to transfer data in wireless sensor networks without using any routing algorithms. In flooding mechanism, each and every sensor node receives data and broadcasts it to all its neighbors till the data is not received at the destination or gets the maximum number of hops to reach data at destination. Gossiping is the improve version of the flooding mechanism where sensor nodes send data to a random neighbor instead of all neighbors.

\subsubsection{Sensor Protocols for Information via Negotiation (SPIN)}

Wendi et.al [1999] proposed a data centric routing protocol called a Sensor Protocol for Information via Negotiation (SPIN). This protocol uses meta-data, before transmitting the data to the sensor node and exchanges meta-data among sensors with the help of broadcast mechanism. [18] Each node which wants to receive new data, advertises it to its neighbors. SPIN's meta-data negotiation assures that there is no redundant data sent throughout the network i.e. it solves the problem of flooding and jamming attack and thus achieves energy efficiency. SPIN exchanges three messages among nodes. These messages are ADV, REQ and DATA to communicate. ADV-is new data advertisement. When any node in the network wants to transfer some information, that node will advertise by transmitting an ADV message which contains the meta-data. REQ-is request for data. If any neighboring node wants to receive the data then it sends REQ message. DATA- is data message; the data message is a collection of actual data (i.e. taken by sensor node) and Meta data header.

\subsubsection{Cougar}

The cougar [5] routing protocol is a based on database approach to tasking sensor networks. This approach provides a user and application programs with declarative queries of the sensed data generated by the source sensors. These queries are suited for WSNs in that they abstract the user from knowing the plan of execution of its queries. In other words, the user does not know which sensors are contacted, how the computation of query is processed of the sensed data, and how final results are sent to the user. The Cougar approach uses a layer of query where each sensor is associated with a query proxy that lies between the network and application layer of the sensor. This query proxy provides higher level services through queries that can be issued from a gateway node. Furthermore, the Cougar approach employs in-network processing to minimize the consumption of total energy and increase the network lifetime. .Cougar is more beneficial if a set of sensed data could be aggregated or fused into a single one that is more representative and thus significant to the user. The cougar being database approach, it faces limited challenges. A network can be viewed as a large distributed database stem, where each sensor possesses a subset of data. Hence, current distributed management approaches cannot be applied directly, but need to be modified accordingly.

\subsubsection{Active Query Forwarding in Sensor Networks (ACQUIRE)}

ACQUIRE [6] is data centric querying mechanism used for querying named data. It gives superior query Optimization to answer specific types of queries, called one-shot complex queries for duplicate data. ACQUIRE query consists of many sub queries for which many simple responses are provided by many relevant sensors. Each and every sub-query is answered Based on the currently stored data at its proper sensor. ACQUIRE allows a sensor to inject an Active query in a network following either a different or a specified trajectory until the query gets Answered by few sensors on the path using a local update mechanism. Unlike other query Techniques, ACQUIRE provides the queries to inject a very complex query into the network to be Forwarded stepwise through a sequence of sensors.

\subsubsection{Gradient Based Routing-Routing on Finger Print Gradient in Sensor Networks (RUGGED)}

RUGGED proposes a novel method to used natural information gradient repository, which is a consequence of the fingerprint gradients of the events effect. It is a totally distributed and reactive routing protocol for WSN. There are much path controlling and exploration instantiation of paths by simulated makes one protocol well suited for high range 


\section{International Journal of Science and Research (IJSR) \\ ISSN (Online): 2319-7064}

Index Copernicus Value (2013): 6.14 | Impact Factor (2015): 6.391

of applications including time gradient based target tracking, event boundary detection.

\subsection{Hierarchical Protocol}

This routing is mainly proposed for the wired type network because this type of routing is best for scalability purpose and it also has a good communication statistics. This type of routing is used the maximum energy nodes. For the aggregation of the data and the remaining nodes are used to collect the data by sensing the environment and sends this data to maximum energy nodes [7]. So the generation of the clusters and the cluster-heads is beneficial for the scalability, and due to this we can enhance the life time of the whole network by using the efficient communication.

\subsubsection{Low-Energy Adoptive Clustering Hierarchy (LEACH)}

Heinzelman et al. [8] who proposed a protocol which is based on the hierarchical routing known as LEACH. It is a clustering based protocol in which some clusters are formed and within the cluster the nodes will be chosen cluster head. The selection of cluster head is a random process and cluster heads are rotated within each round of data processing. Due to the cluster head randomization, cluster heads energy will be well distributed within the each node of the sensor network. It uses the localized coordination which enhances the scalability and fault tolerance. According to the author cluster also changes after some given time interval and hence it creates a uniform energy dissipation within the whole network. The whole operation of the leach protocol will be done in two steps: The Setup phase and The Steady State phase. Under the Setup phase clustering and random selection of cluster head by node within the cluster will be done. And in the steady state phase data transmission from node to cluster head and cluster head to base station will be done.

\subsubsection{TL-LEACH Protocol}

Two Level LEACH protocol is an enhanced version of LEACH [Loscri et al., 2005]. In this new protocol cluster head transfers data to the base station with the help of intermediate node rather than by the direct communication between cluster head to the base station. This technique saves more energy when the distance between cluster head and base station is comparatively more.

\subsubsection{M-LEACH Protocol}

Multi-hop LEACH Protocol, we use multi-hop data transmission between cluster head to the base station. We select optimal path between the cluster head and base station through other cluster heads and use these cluster heads as a relay station to transmit data by them.

\subsubsection{Power-Efficient Gathering in Sensor Information System (PEGASIS)}

In wireless sensor network, another protocol is proposed by Lindsey and Raghavendra [16], which is an improved leach protocol. The methodology behind this protocol is, the node which wants to send the data to the base station, first of all send the data to its neighbor node which is closest to that node and which take part to send the data to the base station. The entire node does same and further a new round is started by this process sensor node reduces the data transmission energy and power will be well distributed in all of the sensor nodes.

PEGASIS have the two main advantages:-

1) The life time of the network will be enhanced by the participation of the entire nodes on the routing.

2) Only local nodes used for the process so the consumption of the bandwidth is very less.

\subsubsection{Threshold-Sensitive Energy-Efficient Protocols (TEEN and APTEEN)}

These protocols TEEN [9] \& Adaptive TEEN [10] are used for the real time applications or for real time data packet. Real time data packets have some time limitation, data sending within certain time period. According to the TEEN protocol, The Sensor node senses the physical medium continuously, but sending of the data is less frequent. Cluster head sending a hard threshold to entire sensor node within the cluster, it is called the attribute for the sensed data. It will also send the soft threshold, which can change the sense attribute and also trigger the particular node to turn on its transmitter and data sending process will start. Hence hard threshold is used to make the less frequent data transmission of the sensed data and data transmission start when the sensed attribute is within the range of our interest.

When the hard threshold getting no change then the number of the transmission by the soft Threshold will be reduced. If the soft threshold is small then the accurate data transmission occurs and by this way the energy consumption of the whole network increases.

\subsubsection{Hybrid Energy-Efficient Distributed Clustering (HEED)}

HEED [11, 12] prolongs the basic scheme of LEACH by using extra energy and node degree or density as a metric for cluster selection to achieve power balancing. It is operates in multi-hop networks, using an adaptive transmission power in the inter-clustering communication. HEED was introduced with four primary goals name are (i) improve network lifetime by distributing energy consumption, (ii) terminating the clustering process within a fixed number of iterations, (iii) minimization of control over head, and (iv) producing very well-distributed $\mathrm{CHs}$ and compressed clusters. In HEED, the proposed algorithm systematically selects $\mathrm{CHs}$ according to a combination of two clustering parameters. The main parameter is their extra energy of each sensor node (used in calculating probability of becoming a $\mathrm{CH}$ ) and the secondary parameter is the intra-cluster cost of communication as a function of node degree or cluster density (i.e. number of neighbors). The main parameter is used to probabilistically select an initial set of $\mathrm{CHs}$ while the secondary parameter is used for breaking ties. The HEED clustering increase network lifetime over LEACH clustering because LEACH randomly selects $\mathrm{CHs}$ (and hence cluster size), which may result in faster death of few nodes. The final CHs selected in HEED are very well distributed across the network and the cost of communication is minimized. However, the cluster selection deals with only a subset of parameters, which can possibly impose constraints on the system. These methods are suitable for improve the network life time rather than for the entire needs of WSN. 


\section{International Journal of Science and Research (IJSR)}

ISSN (Online): 2319-7064

Index Copernicus Value (2013): 6.14 | Impact Factor (2015): 6.391

\subsubsection{Selective Election Protocols (SEP)}

In the SEP protocol the strong nodes (which have the extra energy) is more frequent become cluster head than the normal nodes. Due to this, network life time increases. The stability period also increases and the cluster is balanced when first node dies as well as large amount of sensor motes are dead. One of the problems in the SEP protocol is its direct transmission method, where the cluster head takes data to the remaining nodes within the cluster and sends it to the base station directly. In this process the cluster-head which are far away to the base station will consume more energy and are dying very frequently. Whereas cluster-heads which are near to the base station takes operation until end, that will cause network instability and the network life time is greatly affected.

\subsubsection{An Energy Aware Multi-hop Multi-path Hierarchical (EAMMH)}

Having induced the features of energy aware routing and multi-hop intra cluster routing protocol, this protocol was developed. The operation of the EAMMH protocol is divided into rounds where each round begins with a set-up phase, when the clusters are organized, followed by a steady state phase, when data transfers to the base station occur. According to the overview of the protocol initially the number of nodes has been given as input by the user. A multi-hop intra-cluster network has been formed by organizing the sensor nodes into cluster. Multiple paths have been established from each sensor node to the cluster head and provide an energy aware heuristic function to choose the optimal path.

\subsection{Location-Based Protocols}

In wireless sensor networks, nodes are densely deployed in scattered manner and there is no addressing scheme for sensor network such as IP address. In these types of protocol, each sensor node should be equipped with a small low power Global Positioning System (GPS) receiver so that the location of the nodes is directly available by communicating with a satellite. With the help of location information, one can optimize routing in an energy efficient way [20]. In wireless sensor networks, sleeping the sensor node is the best way to save energy. If the location of sensor node is known then the number of sleeping nodes increases and also solves the problem of designing sleep period schedules. Geographic Adaptive Fidelity (GAF), Geographic and Energy Aware Routing (GEAR) Sinchan Roy chowdhury and Minimum Energy Communication Network (MECN) are popular routing protocols in this category.

\subsubsection{Geographic Adaptive Fidelity (GAF)}

GAF is a location based routing protocol for WSN. It is also an energy knowing routing protocol. It works in such a way that, it turns off irrelevant nodes in the network without affecting the level of routing fidelity, this conserves energy. A virtual grid for the area that is to be covered is formed. The cost of packet routing is considered equivalent for nodes associated with the same point on the virtual grid. Such equivalence is exploited in keeping some nodes located in a particular grid area in sleeping state in order to save energy. By doing this the network lifetime is improve as the number of nodes increases. In this protocol there are three states and they are discovery, for finding the neighbors in the grid, active tells that the nodes are participating in routing and sleep when the radio is turned off. When nodes change states from sleeping to active in turns then the load is balanced.GAF keeps the network connected, by keeping a classical node always in active node for each region on its virtual grid. Although GAF is a location based protocol, it can be considered as a hierarchical protocol, where the clusters are based on geographic location.

\subsubsection{Geographic and Energy-Aware Routing (GEAR)}

GEAR [13] is an energy-efficient routing protocol proposed for routing queries to target regions in a sensor field, In GEAR, the sensors are supposed to have localization hardware equipped, for example, a GPS unit or a localization system[14] so that they know their current position. Furthermore, the sensors know of their extra energy as well as the locations and extra energy of each of their neighbors. GEAR uses energy aware heuristics that are based on information of geographical to select sensors to route a packet toward its terminal region. Then, GEAR uses a recursive geographic forwarding algorithm to broadcast the packet inside the target region.

\subsubsection{Bounded Voronoi Greedy Forwarding (BVGF)}

BVGF [15] using by the concept of Voronoi diagram [16] in which the sensors should be know of their geographical positions. In BVGF, a network is modeled by a Voronoi diagram with sites representing the sensors locations. In this type of greedy geographic routing, a sensor node will always forward a packet to the neighbor that has the shortest path to the destination. The sensors eligible for acting as the next hops are the ones whose Voronoi regions are visited by the segment line combine the source and the destination. The BVGF protocol selected as the next hop the neighbor that has the shortest Euclidean distance to the destination among all eligible neighbors. It does not help the sensors deplete their battery power evenly. Each sensor actually has only one next hop to forward its data to the sink. Therefore, any data spreading path between a source sensor and the sink will always has the same chain of the next hops, which will severely suffer from battery power depletion. BVGF does not consider energy as a metric.

\subsubsection{Minimum Energy Communication Networks (MECN):}

MECN [17] is a location based routing protocol. It maintains a minimum energy network for wireless networks by utilizing low power GPS. This protocol can be used for mobile networks but it is best suited for sensor networks. Because sensor networks are not mobile. A master node is included to a minimum power topology for inactive nodes. MECN assumes a master-site as the information sink, which is always the case for sensor networks. MECN identifies a relay region. This region consists of nodes in a neighboring area where transmission through those nodes is more energy efficient than direct transmission. The main idea of MECN is to find a sub-network which will have minimum number of nodes and require less power for transmission between two nodes. MECN consists of two phases, firstly, it constructs a sparse graph or an enclosure graph, by taking positions of a two dimensional plane. This construction requires local computations in the nodes. The enclosure graph contains 


\section{International Journal of Science and Research (IJSR) \\ ISSN (Online): 2319-7064}

Index Copernicus Value (2013): 6.14 | Impact Factor (2015): 6.391

globally optimal links in terms of energy consumption. Secondly, it finds optimal links on the graph using the Belmann Ford shortest path algorithm. MECN is self organizing and dynamically adapts to nodes failure or the deployment of new sensor nodes.

\section{Conclusion}

The main challenges in the design of energy efficient routing protocols for WSNs due to the limited energy resources of sensors. The main objective behind the designing of routing protocol is to keep the sensors operating for as long as possible, thus improvement of the network lifetime. The consumption of energy in the sensors is dominated by data transmission and reception. Therefore, designing of routing protocols for WSNs should be as energy efficient as possible to increase the individual sensor's lifetime and finally the network lifetime.

In this paper, we have surveyed a case of routing protocols by taking into account several classifications, including Data Centric Routing Protocol, Hierarchical Routing Protocol, Location- Base Routing Protocol. For each of these categories, we have discussed a few example protocols.

\section{References}

[1] Comparative Study of Routing Protocols in Wireless Sensor Network, International Journal of Advanced Research in Computer Science and Software Engineering, Volume 2, Issue 9, September 2012.

[2] I.Akyildiz, W.Su, Y.Sankarasubramaniam, and E. Cayirci, "A survey on sensor networks," IEEE Communications Magazine, vol. 40, no. 8, pp. 102-114, August 2002

[3] M. Ilyas and I. Mahgoub. "Handbook of sensor networks: compact wireless and wired sensing systems".

[4] Chandrakasan, Amirtharajah, Cho, Goodman, Konduri, Kulik, Rabiner, and Wang."Design Considerations for Distributed Microsensor Systems," IEEE 1999 Custom Integrated Circuits Conference (CICC), pages 279-286, May 1999.

[5] Y. Yao and J. Gehrke, "The Cougar approach to innetwork query processing in sensor networks",SGIMOD Record, vol. 31, no. 3, Sept. 2002 ,

[6] N. Sadagopan, B. Krishnamachari, and A. Helmy, "The ACQUIRE mechanism for efficient querying in sensor networks", Proceedings SNPA'03, Anchorage, AK, May 2003, pp. 149-155

[7] Q. Li, J. Aslam, and D. Rus, "Hierarchical power-aware routing in sensor networks," Proc. DIMACS Workshop Pervasive Networking, May, 2001.

[8] W. R. Heinzelman, A. P. Chandrakasan, and H. Balakrishnan, "Energy efficient Communication protocol for wireless Microsensor networks," Proceedings of the 33rd Hawaii International Conference on System Sciences (HICSS-33), January 2000.

[9] A. Manjeshwar and D.P. Agarwal, "TEEN: a routing protocol for enhanced efficiency in wireless sensor networks," 1st Int. Workshop Parallel Distributed Computing Issues Wireless Networks Mobile Computing, April 2001.
[10] A. Manjeshwar and D.P. Agarwal, "APTEEN: a hybrid protocol for efficient routing and comprehensive information retrieval in wireless sensor networks," Parallel Distributed Process. Symp., Proc. Int., IPDPS 2002, 195-202.

[11] Ossama Younis and Sonia Fahmy, "Distributed Clustering in Ad-hoc Sensor Networks: A Hybrid, Energy-efficient Approach", September 2002

[12] Ossama Younis and Sonia Fahmy" Heed: A hybrid, Energy-efficient, Distributed Clustering Approach for Ad-hoc Networks", IEEE Transactions on Mobile Computing, vol. 3, no. 4, Oct.-Dec. 2004, pp.366-369.

[13] Y. Yu, R. Govindan, and D. Estrin, "Geographical and energy aware routing: A recursive data dissemination protocol for wireless sensor networks", Technical Report UCLA /CSD-TR-01-0023, UCLA Computer Science Department, May 2001.

[14] N. Bulusu, J. Heidemann, and D. Estrin, "GPS-less Low Cost Outdoor Localization for Very Small Devices", IEEE Personal Communication Magazine, vol. 7, no. 5, Oct. 2000, pp. 28-34.

[15] G. Xing, C. Lu, R. Pless, and Q. Huang, "On greedy geographic routing algorithms in sensing-covered networks", Proceedings ACM MobiHoc'04, Tokyo, Japan, May 2004, pp. 31-42.

[16]M. Stemm and R. H. Katz, "Measuring and reducing energy consumption of network rfaces in hand- held devices", IEICE Transaction on Communications, vol. E80-B, 8, Aug.1997, pp. 1125-1131

[17] ] V. Rodoplu and T. H. Meng, "Minimum energy mobile wireless networks", IEEE Journal on SelectedAreas in Communications, vol. 17, no. 8, Aug. 1999, pp. 13331344.

[18]Energy Saving Routing Algorithm Based on SPIN Protocol in WSN-Luwei Jing, and Feng Liu College of Science, Huazhong Agricultural University, Wuhan, 430070, Chin, Yuling Li College of Information Engineering, Chutian University of Huazhong Agricultural University,Wuhan, 430205, China-2011

[19] C. Intanagonwiwat, R. Govindan, and D. Estrin, "Directed diffusion for wireless sensor networks," IEEE/ACM Trans. Networking, 11(1), 2-16, 2003

[20] A survey of location based routing protocols for WSN, International Journal of Emerging Technology and Advanced Engineering, Volume 3, Issue 9, September 2013 